\title{
Neighborhoods and partial sums of certain subclass of starlike functions
}

\section{Zhi-Gang Wang*, Xin-Sheng Yuan and Lei Shi}

\section{"Correspondence:}

zhigangwang@foxmail.com

School of Mathematics and

Statistics, Anyang Normal University,

Anyang, Henan 455002, P.R. China

\section{Abstract}

The main purpose of the present paper is to derive the neighborhoods and partial sums of a certain subclass of starlike functions.

MSC: Primary 30C45

Keywords: analytic functions; starlike functions; neighborhoods; partial sums

\section{Introduction}

Let $\mathcal{A}_{m}$ denote the class of functions $f$ of the form

$$
f(z)=z+\sum_{k=m+1}^{\infty} a_{k} z^{k} \quad(m \in \mathbb{N}:=\{1,2,3, \ldots\})
$$

which are analytic in the open unit disk

$$
\mathbb{U}:=\{z: z \in \mathbb{C} \text { and }|z|<1\} .
$$

A function $f \in \mathcal{A}_{m}$ is said to be in the class $\mathcal{S}_{m}^{*}(\beta)$ of starlike functions of order $\beta$ if it satisfies the inequality

$$
\Re\left(\frac{z f^{\prime}(z)}{f(z)}\right)>\beta \quad(z \in \mathbb{U} ; 0 \leqq \beta<1) .
$$

Assuming that $\alpha \geqq 0,0 \leqq \beta<1$ and $f \in \mathcal{A}_{m}$, we say that a function $f \in \mathcal{H}_{m}(\alpha, \beta)$ if it satisfies the condition

$$
\Re\left(\frac{z f^{\prime}(z)}{f(z)}+\alpha \frac{z^{2} f^{\prime \prime}(z)}{f(z)}\right)>\alpha \beta\left(\beta+\frac{m}{2}-1\right)+\beta-\frac{m \alpha}{2} \quad(z \in \mathbb{U}) .
$$

For convenience, throughout this paper, we write

$$
\gamma_{m}:=\alpha \beta\left(\beta+\frac{m}{2}-1\right)+\beta-\frac{m \alpha}{2} .
$$

Recently, Ravichandran et al. [1] proved that $\mathcal{H}_{m}(\alpha, \beta) \subset \mathcal{S}_{m}^{*}(\beta)$. Subsequently, Liu et $a l$. [2] derived various properties and characteristics such as inclusion relationships, Hadamard products, coefficient estimates, distortion theorems and cover theorems for

\section{Springer}

(c) 2013 Wang et al.; licensee Springer. This is an Open Access article distributed under the terms of the Creative Commons Attribution License (http://creativecommons.org/licenses/by/2.0), which permits unrestricted use, distribution, and reproduction in any medium, provided the original work is properly cited. 
the class $\mathcal{H}_{m}(\alpha, \beta)$ and a subclass of $\mathcal{H}_{m}(\alpha, \beta)$ with negative coefficients. Furthermore, Singh et al. [3] generalized the class $\mathcal{H}_{m}(\alpha, \beta)$ and found several sufficient conditions for starlikeness. In the present paper, we aim at proving the neighborhoods and partial sums of the class $\mathcal{H}_{m}(\alpha, \beta)$.

\section{Main results}

Following the earlier works (based upon the familiar concept of a neighborhood of analytic functions) by Goodman [4] and Ruscheweyh [5], and (more recently) by Altintaş et al. [6-9], Cătaş [10], Frasin [11], Keerthi et al. [12] and Srivastava et al. [13], we begin by introducing here the $\delta$-neighborhood of a function $f \in \mathcal{A}_{m}$ of the form (1.1) by means of the definition

$$
\begin{aligned}
\mathcal{N}_{\delta}(f):= & \left\{g \in \mathcal{A}_{m}: g(z)=z+\sum_{k=m+1}^{\infty} b_{k} z^{k}\right. \text { and } \\
& \left.\sum_{k=m+1}^{\infty} \frac{k(1+k \alpha-\alpha)-\gamma_{m}}{1-\gamma_{m}}\left|a_{k}-b_{k}\right| \leqq \delta\left(\delta, \alpha \geqq 0 ; 0 \leqq \beta<1 ; \gamma_{m}<1\right)\right\} .
\end{aligned}
$$

By making use of the definition (2.1), we now derive the following result.

Theorem 1 Iff $\in \mathcal{A}_{m}$ satisfies the condition

$$
\frac{f(z)+\varepsilon z}{1+\varepsilon} \in \mathcal{H}_{m}(\alpha, \beta) \quad(\varepsilon \in \mathbb{C} ;|\varepsilon|<\delta ; \delta>0)
$$

then

$$
\mathcal{N}_{\delta}(f) \subset \mathcal{H}_{m}(\alpha, \beta)
$$

Proof By noting that the condition (1.3) can be rewritten as follows:

$$
\left|\frac{\frac{z f^{\prime}(z)}{f(z)}+\alpha \frac{z^{2} f^{\prime \prime}(z)}{f(z)}-1}{\frac{z f^{\prime}(z)}{f(z)}+\alpha \frac{z^{2} f^{\prime \prime}(z)}{f(z)}-\left(2 \gamma_{m}-1\right)}\right|<1 \quad(z \in \mathbb{U})
$$

we easily find from (2.4) that a function $g \in \mathcal{H}_{m}(\alpha, \beta)$ if and only if

$$
\frac{z g^{\prime}(z)+\alpha z^{2} g^{\prime \prime}(z)-g(z)}{z g^{\prime}(z)+\alpha z^{2} g^{\prime \prime}(z)-\left(2 \gamma_{m}-1\right) g(z)} \neq \sigma \quad(z \in \mathbb{U} ; \sigma \in \mathbb{C} ;|\sigma|=1)
$$

which is equivalent to

$$
\frac{(g * h)(z)}{z} \neq 0 \quad(z \in \mathbb{U}),
$$

where

$$
h(z)=z+\sum_{k=m+1}^{\infty} c_{k} z^{k} \quad\left(c_{k}:=\frac{k+\alpha k(k-1)-1-\left[k+\alpha k(k-1)-\left(2 \gamma_{m}-1\right)\right] \sigma}{2\left(\gamma_{m}-1\right) \sigma}\right) .
$$


It follows from (2.6) that

$$
\begin{aligned}
\left|c_{k}\right| & =\left|\frac{k+\alpha k(k-1)-1-\left[k+\alpha k(k-1)-\left(2 \gamma_{m}-1\right)\right] \sigma}{2\left(\gamma_{m}-1\right) \sigma}\right| \\
& \leqq \frac{k+\alpha k(k-1)-1+\left[k+\alpha k(k-1)-\left(2 \gamma_{m}-1\right)\right]|\sigma|}{2\left(1-\gamma_{m}\right)|\sigma|} \\
& =\frac{k(1+k \alpha-\alpha)-\gamma_{m}}{1-\gamma_{m}} \quad(|\sigma|=1) .
\end{aligned}
$$

If $f \in \mathcal{A}_{m}$ satisfies the condition (2.2), we deduce from (2.5) that

$$
\frac{(f * h)(z)}{z} \neq-\varepsilon \quad(|\varepsilon|<\delta ; \delta>0)
$$

or, equivalently,

$$
\left|\frac{(f * h)(z)}{z}\right| \geqq \delta \quad(z \in \mathbb{U} ; \delta>0) .
$$

We now suppose that

$$
q(z)=z+\sum_{k=m+1}^{\infty} d_{k} z^{k} \in \mathcal{N}_{\delta}(f)
$$

It follows from (2.1) that

$$
\begin{aligned}
\left|\frac{((q-f) * h)(z)}{z}\right| & =\left|\sum_{k=m+1}^{\infty}\left(d_{k}-a_{k}\right) c_{k} z^{k-1}\right| \\
& \leqq|z| \sum_{k=m+1}^{\infty} \frac{k(1+k \alpha-\alpha)-\gamma_{m}}{1-\gamma_{m}}\left|d_{k}-a_{k}\right|<\delta .
\end{aligned}
$$

Combining (2.7) and (2.8), we easily find that

$$
\left|\frac{(q * h)(z)}{z}\right|=\left|\frac{([f+(q-f)] * h)(z)}{z}\right| \geqq\left|\frac{(f * h)(z)}{z}\right|-\left|\frac{((q-f) * h)(z)}{z}\right|>0,
$$

which implies that

$$
\frac{(q * h)(z)}{z} \neq 0 \quad(z \in \mathbb{U}) .
$$

Therefore, we conclude that

$$
q(z) \in \mathcal{N}_{\delta}(f) \subset \mathcal{H}_{m}(\alpha, \beta)
$$

We thus complete the proof of Theorem 1.

Next, we derive the partial sums of the class $\mathcal{H}_{m}(\alpha, \beta)$. For some recent investigations involving the partial sums in analytic function theory, one can refer to [14-16] and the references cited therein. 
Theorem 2 Let $f \in \mathcal{A}_{m}$ be given by (1.1) and define the partial sums $f_{n}(z)$ off by

$$
f_{n}(z)=z+\sum_{k=m+1}^{n} a_{k} z^{k} \quad(n \in \mathbb{N} ; n \geqq m+1) .
$$

If

$$
\sum_{k=m+1}^{\infty} \frac{k(1+k \alpha-\alpha)-\gamma_{m}}{1-\gamma_{m}}\left|a_{k}\right| \leqq 1 \quad\left(\alpha \geqq 0 ; 0 \leqq \beta<1 ; \gamma_{m}<1\right),
$$

then

(1) $f \in \mathcal{H}_{m}(\alpha, \beta)$;

(2)

$$
\Re\left(\frac{f(z)}{f_{n}(z)}\right) \geqq \frac{n(1+\alpha+n \alpha)}{(n+1)(1+n \alpha)-\gamma_{m}} \quad(n \in \mathbb{N} ; n \geqq m+1 ; z \in \mathbb{U})
$$

and

$$
\Re\left(\frac{f_{n}(z)}{f(z)}\right) \geqq \frac{(n+1)(1+n \alpha)-\gamma_{m}}{(n+1)(1+n \alpha)+1-2 \gamma_{m}} \quad(n \in \mathbb{N} ; n \geqq m+1 ; z \in \mathbb{U}) .
$$

The bounds in (2.11) and (2.12) are sharp.

Proof (1) Suppose that $f_{1}(z)=z$. We know that $z \in \mathcal{H}_{m}(\alpha, \beta)$, which implies that

$$
\frac{f_{1}(z)+\varepsilon z}{1+\varepsilon}=z \in \mathcal{H}_{m}(\alpha, \beta) .
$$

From (2.10), we easily find that

$$
\sum_{k=m+1}^{\infty} \frac{k(1+k \alpha-\alpha)-\gamma_{m}}{1-\gamma_{m}}\left|a_{k}-0\right| \leqq 1
$$

which implies that $f \in \mathcal{N}_{1}(z)$. In view of Theorem 1 , we deduce that

$$
f \in \mathcal{N}_{1}(z) \subset \mathcal{H}_{m}(\alpha, \beta) .
$$

(2) It is easy to verify that

$$
\begin{aligned}
\frac{(n+1)[1+(n+1) \alpha-\alpha]-\gamma_{m}}{1-\gamma_{m}} & =\frac{(n+1)(1+n \alpha)-\gamma_{m}}{1-\gamma_{m}} \\
& >\frac{n(1+n \alpha-\alpha)-\gamma_{m}}{1-\gamma_{m}}>1 \quad(n \in \mathbb{N}) .
\end{aligned}
$$

Therefore, we have

$$
\sum_{k=m+1}^{n}\left|a_{k}\right|+\frac{(n+1)(1+n \alpha)-\gamma_{m}}{1-\gamma_{m}} \sum_{k=n+1}^{\infty}\left|a_{k}\right| \leqq \sum_{k=m+1}^{\infty} \frac{k(1+k \alpha-\alpha)-\gamma_{m}}{1-\gamma_{m}}\left|a_{k}\right| \leqq 1
$$


We now suppose that

$$
\begin{aligned}
\psi(z) & =\frac{(n+1)(1+n \alpha)-\gamma_{m}}{1-\gamma_{m}}\left(\frac{f(z)}{f_{n}(z)}-\frac{n(1+\alpha+n \alpha)}{(n+1)(1+n \alpha)-\gamma_{m}}\right) \\
& =1+\frac{\frac{(n+1)(1+n \alpha)-\gamma_{m}}{1-\gamma_{m}} \sum_{k=n+1}^{\infty} a_{k} z^{k-1}}{1+\sum_{k=m+1}^{n} a_{k} z^{k-1}} .
\end{aligned}
$$

It follows from (2.13) and (2.14) that

$$
\left|\frac{\psi(z)-1}{\psi(z)+1}\right| \leqq \frac{\frac{(n+1)(1+n \alpha)-\gamma_{m}}{1-\gamma_{m}} \sum_{k=n+1}^{\infty}\left|a_{k}\right|}{2-2 \sum_{k=m+1}^{n}\left|a_{k}\right|-\frac{(n+1)(1+n \alpha)-\gamma_{m}}{1-\gamma_{m}} \sum_{k=n+1}^{\infty}\left|a_{k}\right|} \leqq 1 \quad(z \in \mathbb{U}),
$$

which shows that

$$
\Re(\psi(z)) \geqq 0 \quad(z \in \mathbb{U}) .
$$

Combining (2.14) and (2.15), we deduce that the assertion (2.11) holds true.

Moreover, if we put

$$
f(z)=z+\frac{1-\gamma_{m}}{(n+1)(1+n \alpha)-\gamma_{m}} z^{n+1} \quad(n \in \mathbb{N} \backslash\{1,2, \ldots, m-1\} ; m \in \mathbb{N}),
$$

then for $z=r e^{i \pi / n}$, we have

$$
\frac{f(z)}{f_{n}(z)}=1+\frac{1-\gamma_{m}}{(n+1)(1+n \alpha)-\gamma_{m}} z^{n} \rightarrow \frac{n(1+\alpha+n \alpha)}{(n+1)(1+n \alpha)-\gamma_{m}} \quad\left(r \rightarrow 1^{-}\right),
$$

which implies that the bound in (2.11) is the best possible for each $n \in \mathbb{N} \backslash\{1,2, \ldots, m-1\}$.

Similarly, we suppose that

$$
\begin{aligned}
\varphi(z) & =\frac{(n+1)(1+n \alpha)+1-2 \gamma_{m}}{1-\gamma_{m}}\left(\frac{f_{n}(z)}{f(z)}-\frac{(n+1)(1+n \alpha)-\gamma_{m}}{(n+1)(1+n \alpha)+1-2 \gamma_{m}}\right) \\
& =1-\frac{\frac{(n+1)(1+n \alpha)+1-2 \gamma_{m}}{1-\gamma_{m}} \sum_{k=n+1}^{\infty} a_{k} z^{k-1}}{1+\sum_{k=m+1}^{\infty} a_{k} z^{k-1}} .
\end{aligned}
$$

In view of (2.13) and (2.17), we conclude that

$$
\left|\frac{\varphi(z)-1}{\varphi(z)+1}\right| \leqq \frac{\frac{(n+1)(1+n \alpha)+1-2 \gamma_{m}}{1-\gamma_{m}} \sum_{k=n+1}^{\infty}\left|a_{k}\right|}{2-2 \sum_{k=m+1}^{n}\left|a_{k}\right|-\frac{n(1+\alpha+n \alpha)}{1-\gamma_{m}} \sum_{k=n+1}^{\infty}\left|a_{k}\right|} \leqq 1 \quad(z \in \mathbb{U}),
$$

which implies that

$$
\Re(\varphi(z)) \geqq 0 \quad(z \in \mathbb{U})
$$

Combining (2.17) and (2.18), we readily get the assertion (2.12) of Theorem 2 . The bound in (2.12) is sharp with the extremal function $f$ given by (2.16).

The proof of Theorem 2 is thus completed. 
Finally, we turn to ratios involving derivatives. The proof of Theorem 3 below is much akin to that of Theorem 2, we here choose to omit the analogous details.

Theorem 3 Let $f \in \mathcal{A}_{m}$ be given by (1.1) and define the partial sums $f_{n}(z)$ of $f$ by (2.9). If the condition (2.10) holds, then

$$
\Re\left(\frac{f^{\prime}(z)}{f_{n}^{\prime}(z)}\right) \geqq \frac{(n+1)\left(n \alpha+\gamma_{m}\right)-\gamma_{m}}{(n+1)(1+n \alpha)-\gamma_{m}} \quad(n \in \mathbb{N} ; n \geqq m+1 ; z \in \mathbb{U})
$$

and

$$
\Re\left(\frac{f_{n}^{\prime}(z)}{f^{\prime}(z)}\right) \geqq \frac{(n+1)(1+n \alpha)-\gamma_{m}}{(n+1)\left(2+n \alpha-\gamma_{m}\right)-\gamma_{m}} \quad(n \in \mathbb{N} ; n \geqq m+1 ; z \in \mathbb{U}) .
$$

The bounds in (2.19) and (2.20) are sharp with the extremal function given by (2.16).

Remark By setting $\alpha=0$ and $m=1$ in Theorems 2 and 3, we get the corresponding results obtained by Silverman [16].

\section{Competing interests}

The authors declare that they have no competing interests.

\section{Authors' contributions}

The authors completed the paper together. They also read and approved the final manuscript.

\section{Acknowledgements}

Dedicated to Professor Hari M Srivastava.

The present investigation was supported by the National Natural Science Foundation under Grants 11226088 and 11101053, the Key Project of Chinese Ministry of Education under Grant 211118, the Excellent Youth Foundation of Educational Committee of Hunan Province under Grant 10B002, the Open Fund Project of Key Research Institute of Philosophies and Social Sciences in Hunan Universities under Grants 11 FEFM02 and 12FEFM02, and the Key Project of Natural Science Foundation of Educational Committee of Henan Province under Grant 12A110002 of the People's Republic of China.

Received: 7 December 2012 Accepted: 8 March 2013 Published: 10 April 2013

\section{References}

1. Ravichandran, V, Selvaraj, C, Rajalaksmi, R: Sufficient conditions for starlike functions of order $\alpha$. J. Inequal. Pure Appl. Math. 3, Article ID 81 (2002) (electronic)

2. Liu, M-S, Zhu, Y-C, Srivastava, HM: Properties and characteristics of certain subclasses of starlike functions of order $\beta$. Math. Comput. Model. 48, 402-419 (2008)

3. Singh, S, Gupta, S, Singh, S: Starlikeness of analytic maps satisfying a differential inequality. Gen. Math. 18, 51-58 (2010)

4. Goodman, AW: Univalent functions and nonanalytic curves. Proc. Am. Math. Soc. 8, 598-601 (1957)

5. Ruscheweyh, S: Neighborhoods of univalent functions. Proc. Am. Math. Soc. 81, 521-527 (1981)

6. Altintaş, O: Neighborhoods of certain $p$-valently analytic functions with negative coefficients. Appl. Math. Comput. 187, 47-53 (2007)

7. Altintaş, O, Owa, S: Neighborhoods of certain analytic functions with negative coefficients. Int. J. Math. Math. Sci. 19, 797-800 (1996)

8. Altintaş, O, Özkan, Ö, Srivastava, HM: Neighborhoods of a class of analytic functions with negative coefficients. Appl. Math. Lett. 13, 63-67 (2000)

9. Altintaş, O, Özkan, Ö, Srivastava, HM: Neighborhoods of a certain family of multivalent functions with negative coefficients. Comput. Math. Appl. 47, 1667-1672 (2004)

10. Cătaş, A: Neighborhoods of a certain class of analytic functions with negative coefficients. Banach J. Math. Anal. 3 , 111-121 (2009)

11. Frasin, BA: Neighborhoods of certain multivalent functions with negative coefficients. Appl. Math. Comput. 193, 1-6 (2007)

12. Keerthi, BS, Gangadharan, A, Srivastava, HM: Neighborhoods of certain subclasses of analytic functions of complex order with negative coefficients. Math. Comput. Model. 47, 271-277 (2008)

13. Srivastava, HM, Eker, SS, Seker, B: Inclusion and neighborhood properties for certain classes of multivalently analytic functions of complex order associated with the convolution structure. Appl. Math. Comput. 212, 66-71 (2009)

14. Frasin, BA: Partial sums of certain analytic and univalent functions. Acta Math. Acad. Paedagog. Nyházi. 21, 135-145 (2005) (electronic) 
15. Frasin, BA: Generalization of partial sums of certain analytic and univalent functions. Appl. Math. Lett. 21, 735-741 (2008)

16. Silverman, H: Partial sums of starlike and convex functions. J. Math. Anal. Appl. 209, 221-227 (1997)

doi:10.1186/1029-242X-2013-163

Cite this article as: Wang et al.: Neighborhoods and partial sums of certain subclass of starlike functions. Journal of Inequalities and Applications 2013 2013:163.

Submit your manuscript to a SpringerOpen ${ }^{\circ}$ journal and benefit from:

- Convenient online submission

Rigorous peer review

- Immediate publication on acceptance

- Open access: articles freely available online

- High visibility within the field

- Retaining the copyright to your article

Submit your next manuscript at $>$ springeropen.com 